\title{
ASYMPTOTIC COMPLETENESS OF SHORT-RANGE MANY-BODY SYSTEMS ${ }^{1}$
}

\author{
BY I. M. SIGAL AND A. SOFFER ${ }^{2}$
}

\begin{abstract}
We announce a proof of asymptotic completeness for quantum mechanical systems consisting of arbitrary numbers of particles interacting via short-range forces (see conditions (A)-(D) below). A complete proof is given in [SigSof 1]. Previously, there were only partial results in this direction (see reviews and discussions in [Enss, RSIII, Sig]).
\end{abstract}

Consider an $N$-body system in the physical space $\mathbf{R}^{\nu}$. The configuration space in the center-of-mass frame is $X=\left\{x \in \mathbf{R}^{\nu N} \mid \sum m_{i} x_{i}=0\right\}$ with the inner product $\langle x, y\rangle=2 \sum m_{i} x_{i} \cdot y_{i}$. Here $m_{i}>0$ are masses of the particles in question. The Schrödinger operator of such a system is

$$
H=-\Delta+V(x) \text { on } L^{2}(X) \text {. }
$$

Here $\Delta$ is the Laplacian on $X$ and $V(x)=\sum V_{i j}\left(x_{i}-x_{j}\right)$. We assume that the potentials $V_{i j}$ are real and obey (with $\langle x\rangle=\left(1+|x|^{2}\right)^{1 / 2}$ )

(A) $V_{i j}(y)$ are $\Delta_{y}$-compact,

(B) $\langle y\rangle^{1+\theta}\left|\nabla V_{i j}(y)\right|$ are $\Delta_{y}$-bounded for some $\theta>0$,

(C) $|y|^{2} \Delta V_{i j}(y)$ are $\Delta_{y}$-bounded,

(D) $\langle y\rangle^{\mu} V_{i j}(y)$ are $\Delta_{y}$-bounded.

Due to condition (A), $H$ is selfadjoint on $D(H)=D(\Delta)$ by the Kato theorem (see e.g. [RSII]). By a short-range system we understand a system obeying (A) and (D) with $\mu>1$. Our main result is

THEOREM 1. Assume an $N$-body system is described by potentials obeying conditions (A)-(D) with $\mu>1$. Then asymptotic completeness holds for this system. tion.

Conditions (B)-(D) can be relaxed if we exercise more care in our estima-

Denote by $a, b, \ldots$, partitions of the set $\{1, \ldots, N\}$ into nonempty disjoint subsets, called clusters. The relation $b \subset a$ means that $b$ is a refinement of $a$. We assume that partitions have at least two clusters. We define the intercluster interaction for a partition $a$ as

$$
I_{a}=\text { sum of all potentials linking different clusters in } a,
$$

and the Hamiltonian for the system composed of the noninteracting clusters: $H_{a}=H-I_{a}$. Furthermore, $H^{a}$ stands for the Hamiltonian of the same

Received by the editors August 20, 1985.

1980 Mathematics Subject Classification (1985 Revision). Primary 81F10; Secondary 81H05, 35P25, 47A40.

${ }^{1}$ Research partially supported by NSF.

${ }^{2} \mathrm{~A}$. Soffer is a Weizmann Fellow in Mathematics and Physics. 
system but with the centers-of-mass of the clusters fixed at the origin. These operators are, clearly, selfadjoint.

An $N$-body short-range system described by a Hamiltonian $H$ is said to be asymptotically complete if for any $\psi$ orthogonal to the eigenfunctions of $H$ there exist Hilbert space vectors $\psi_{a}^{ \pm}$s.t.

$$
\left\|e^{-i H t} \psi-\sum_{a} e^{-i H_{a} t} \psi_{a}^{ \pm}\right\| \rightarrow 0 \quad \text { as } t \rightarrow \pm \infty
$$

and the corresponding statements hold for any of its subsystems (see [Enss, RSIII, Sig] for different, equivalent definitions).

The feature which distinguishes an $N$-body Schrödinger operator from a two-body one, creating a different level of complexity, is that in the manybody case the potential $V(x)$ does not vanish as $|x| \rightarrow \infty$ along the planes

$$
X_{a}=\left\{x \in X \mid x_{i}=x_{j} \text { if } i \text { and } j \text { belong to same clusters of } a\right\}
$$

(the configuration spaces of the relative motion of the clusters in decomposition $a$ ). This feature leads to the multichannel structure of the scattering process. Namely, as $t \rightarrow \pm \infty$, the Schrödinger state $\psi_{t}=e^{-i H t} \psi$ approaches a superposition of waves each of which propagates freely along a certain $X_{a}$ and with the Hamiltonian $\left|p_{a}\right|^{2}$, where $p_{a}=-i\left(\operatorname{grad}\right.$ along $\left.X_{a}\right)$, with the motion in the orthogonal plane, $X \ominus X_{a}$, bounded and described by an eigenfunction of the internal Hamiltonian $H^{a}$. Each of these waves represents a different scenario for the scattering process to develop according to. These scenarios are called channels. A measurement always finds a system in question in one of the possible channels. Put differently, since the free motion is asymptotically a classical motion, the above picture describes an evolution of stable quantum clusters which becomes classical as $t \rightarrow \pm \infty$. This mixture of classical and quantum-mechanical behavior is the unique feature of the many-body scattering theory. We contend that there is a (phase-space) tunneling between certain channels (see [SigSof 2]) and it produces a motion in the classically forbidden region for arbitrary large times. This phenomenon occurs only for $N \geq 4$. The fact that asymptotic completeness nevertheless holds is due to a certain self-averaging process (see below), and we expect that the statement of asymptotic completeness cannot be strenghened in an essential way (e.g., by requiring decay of the probabilities as $t^{-\gamma}$ ). This borderline nature is, probably, one of the reasons why the problem eluded a solution for such a long time.

A word about notations. The dual space to $X$ is denoted by $X^{\prime}$, etc. Given generic vectors $x \in X$ and $k \in X^{\prime}$, their projections on $X_{a}$ and $X_{a}^{\prime}$ are denoted by $x_{a}$ and $k_{a}$, respectively. Finally, $p=-i$ grad.

Given $E$, we define the phase-space domains

$$
P S_{E}^{ \pm}=\bigcup_{\lambda, a}\left\{(x, k) \in\left(X_{a} \backslash\left[\bigcup_{b \supsetneq a} X_{b}\right]\right) \times\left. X^{\prime}\left|x_{a} \| k_{a},\right| k_{a}\right|^{2}=E-\lambda\right\},
$$

where $u \| v$ means that the vectors $u$ and $v$ are parallel and we use the agreement that $\sigma_{p}\left(H^{a}\right)=\{0\}$ for $a$, the partition into the $N$ clusters $(1), \ldots,(N)$. 
Here the union extends over all $(\lambda, a)$ satisfying $\lambda \in \sigma_{p}\left(H^{a}\right) \cap(-\infty, E] \neq \varnothing$. $P S_{E}^{ \pm}$describe different possibilities of outgoing/incoming free motion of noninteracting, stable clusters of the total energy $E$. The restriction on their kinetic energy $\left(\left|k_{a}\right|^{2}\right.$, where $a$ is a cluster break-up realized in the process) stems from the energy conservation law and the fact that the internal energy of the stable clusters must be given by an eigenvalue of their internal Hamiltonian $\left(H^{a}\right)$. The next theorem shows that as $t \rightarrow \pm \infty$ the quantum evolution $e^{-i H t} \psi$, with $\psi$ in a small energy interval around $E$, becomes localized in the phase-space region $P S_{E}^{ \pm}$. We begin with some definitions. Operators of the following form appear naturally in our analysis: $\sum j_{i}(x) f_{i}(p)$, where the sum is finite, all functions are smooth and bounded and, in addition, $j_{i}$ are homogeneous of degree 0 for $|x| \geq 1$. Such operators will be called the phase-space operators. $\sum j_{i} f_{i}$ is said to be supported in the phase-space region $\bigcup_{i}\left[\left(\operatorname{supp} j_{i}\right) \times\left(\operatorname{supp} f_{i}\right)\right]$. Given $E$, we say that a subset of the phase space $X \times X^{\prime}$ is a future/past propagation set at the energy $E$ if for any phase-space operator $J$, supported outside of the set in question, there is a small interval $\Delta$ around $E$ s.t. for any $\psi \in \operatorname{Ran} P_{\Delta}(H)$

$$
\pm \int_{0}^{ \pm \infty}\left\|J \frac{1}{\sqrt{\langle x\rangle}} e^{-i H t} \psi\right\|^{2} d t \leq C\|\psi\|^{2},
$$

with $C<\infty$ and independent of $\psi$.

REMARK 2. this definition is equivalent to one in which the phase-space operators are replaced by more general pseudodifferential operators. However, since, as was mentioned above, the phase-space operators arise naturally in our approach we restrict our definitions to such operators. Much more importantly, one can refine the definition of the propagation set by introducing the coefficient of proportionality into the relation $x_{a} \| \pm k_{a}$-the time ([SigSof 2]).

Propagation TheOREM. Let (A)-(C) be satisfied (note: condition (D) is not required). Then $P S_{E}^{ \pm}$is the future/past propagation set at the energy $E$.

To prove this theorem we use positivity estimates of the commutators of $H$ with certain phase-space operators and with functions of the selfadjoint operator $\gamma=\frac{1}{2}(x \cdot p+p \cdot x)$ where $x=x /\langle x\rangle$. Basic to these estimates is a delicate channel expansion of $P_{\Delta}(H) i[H, \gamma] P_{\Delta}(H)$.

Now we proceed to the second ingredient of our approach. We use the standard notation $|x|_{a}=\min \left\{\left|x_{i}-x_{j}\right| \mid i\right.$ and $j$ belong to different clusters of $a\}$. This is the intercluster distance in the decomposition a.

Channel Decoupling Theorem. Given $E$, there are phase-space operators $j_{a}(x, p)$, which form a partition of unity: $\sum j_{a}(x, p)=\mathbf{1}$, with the following properties:

(2) $j_{a}$ are supported in $\left\{(x, k) \in X \times\left. X^{\prime}|| x\right|_{a}>\delta|x|\right\}$ for some $\delta>0$;

(3) $\left[H_{a}, j_{a}\right]=(|p|$-bounded operator $) \times($ phase-space operator supported away from $\left.P S_{E}=P S_{E}^{+} \cup P S_{E}^{-}\right)$. 
Relation (2) shows that $j_{a}$ is supported in the region where the clusters in $a$ move away from each other and relation (3) shows that the "boundary", $\operatorname{supp}\left(\left[H_{a}, j_{a}\right]\right)$, of $j_{a}$ lives in the region where no propagation takes place.

To derive asymptotic completeness (Theorem 1 ) from the propagation and channel decoupling theorems we introduce the Deift-Simon wave operators for given $E$ and sufficiently small $\Delta \ni E$ :

$$
W_{a}^{ \pm}=\underset{t \rightarrow \pm \infty}{\mathrm{s}-\lim } e^{i H_{a} t} j_{a}(x, p) e^{-i H t}
$$

if the limits exist on $\operatorname{Ran} P_{\Delta}(H)$. (Deift and Simon [DS] have introduced such operators for a partition of unity depending only on $x$.) After this, the main steps are as follows.

LEMMA 3 (Deift-Simon argument). If the limits (4) exist on $\operatorname{Ran} P_{\Delta}$ then asymptotic completeness holds on $\Delta$.

Proposition 4. Let (A)-(D), with $\mu>1$, hold and let the channel decoupling and propagation theorems hold. Then the Deift-Simon wave operators $W_{a}^{ \pm}$exist on $\operatorname{Ran} P_{\Delta}$, provided $\Delta$ is disjoint from the thresholds and eigenvalues of $H$.

SKETCH OF THE PROOF. Let $W_{a}(t)=e^{i H_{a} t} j_{a}(x, p) e^{-i H t}$. By the fundamental theorem of calculus

$$
W_{a}(t)=j_{a}+i \int_{0}^{t} e^{i H_{a} s}\left(H_{a} j_{a}-j_{a} H\right) e^{-i H s} d s .
$$

We have $H_{a} j_{a}-j_{a} H=\left[H_{a}, j_{a}\right]-j_{a} I_{a}$. Due to property (2) of $j_{a}$ and condition (D) on the potentials, $j_{a} I_{a}(H+i)^{-1}=O\left(|x|^{-\mu}\right)$. (This is the only place where condition (D) is used!) Then a result of [PSS] (a local $H$-smoothness of $\langle x\rangle^{-(1 / 2)-\varepsilon}$ shows that $j_{a} I_{a}$ yields a convergent contribution to (5). To show that $\left[H_{a}, j_{a}\right]$ gives a convergent contribution to (5) we use the homogeneity of $j_{a}$ in $x$ for $|x| \geq 1$ and the fact that due to (3) $\left[H_{a}, j_{a}\right]$ is supported away from the propagation set $P S_{E}$. The latter fact allows us to use estimates (1) of the propagation theorem.

\section{REFERENCES}

[DS] P. Deift and B. Simon, A time-dependent approach to the completeness of multiparticle quantum systems, Comm. Pure Appl. Math. 30 (1977), 573-583.

[Enss] V. Enss, Proc. Como summer school on the Schrödinger equation, Como, 1984 (to appear).

[PSS] P. Perry, I. M. Sigal and B. Simon, Spectral analysis of N-body Schrödinger operators, Ann. of Math. (2) 144 (1981), 519-567.

[RS] M. Reed and B. Simon, Methods of modern mathematical physics. II, III, Academic Press, 1975, 1979.

[Sig] I. M. Sigal, Scattering theory for many-body quantum mechanical systems, Springer Lecture Notes in Math., vol. 1011, 1983.

[SigSof 1] I. M. Sigal and A. Soffer, preprint.

[SigSof 2] _ , in preparation.

Department of Mathematics, UNiVersity of CALIForNia, IRVINe, CaliFORNIA 92717

Division of Mathematics, Physics and Astronomy, Caltech, Pasadena, CALIFORNIA 91125 